\title{
Weight Calculation for Cases Generated by Tacit Knowledge Explicit Based on RS-FAHP
}

\author{
Yue Cao ${ }^{1, *}$, Zengmao Guo ${ }^{1,2}$ and Jianhua Zhang ${ }^{1}$ \\ ${ }^{1}$ Management Engineering Department, Zhengzhou University, Zhengzhou, China \\ ${ }^{2}$ Henan Electronic Commerce Association, Zhengzhou, China \\ Corresponding Email: 1217200588@qq.com
}

\begin{abstract}
In the knowledge economy, it becomes the core competence of persons, groups and organizations to effectively organize and manage tacit knowledge, affecting their sustainable development. Case explicit for tacit knowledge is an effective way to improve their clarity, improve management efficiency. it determines the validity of the case view to calculate legitimately the weights for the case aspects or attributes, and further affect the application benefit of the explicit knowledge. The case view affected seriously by the subjective, obtaining via traditional direct weighting method, and the objectivity of the result is not strong. On the other hand, the objective weights configuration is not only ignored the expert knowledge, but also lead to the acceptance barriers for the body of knowledge to accept the result. Therefore, in this paper, relying on rough set (RS) theory, the integrating algorithm of two objective weight configuration is analyzed Systematically, based on conditional entropy and property dependence. Simultaneously, Fuzzy Analytic Hierarchy Process (AHP) is studied to take into account the operational experience and knowledge of experts in the field. And then, case attribute RS-FAHP comprehensive weight placement algorithms is designed, based on the integration of subjective and objective thinking. The work mentioned above can improve and perfect the traditional configuration of weights, and support to apply and manage the tacit knowledge explicit cases effectively.
\end{abstract}

\section{Introduction}

In the era of knowledge economy, knowledge evolved into the core of production, become the main source of value creation [1,2].In the main body of knowledge structure, tacit knowledge not only accounts for most of it, but also directly determines the realization of knowledge application and innovation; However, due to its low level of clarity, the organization and management efficiency of tacit knowledge in the domain of practice is relatively low. This restricts the implementation of knowledge management (Knowledge Management, KM) performance. Artificial intelligence based on case-based Reasoning (Case-based Reasoning, CBR) system is a case of tacit knowledge explicit management of self-learning technique, it is based on historical problems and its solutions to assist users to make decisions or solve existing similar problems. So here, the author through the CBR technology implementation of KM tacit knowledge explicit organization and management, to improve efficiency and effectiveness of KM implementation.

In the traditional "4R" cycle of CBR model (Retrieve-Reuse-Revise-Retain), the retrieve is a core activity, its speed and accuracy directly decides the actual level of CBR system. Case retrieval is based on matching calculation, which requires a reasonable and efficient case-based weight vector computing mechanism. There are many traditional weight calculation method, such as direct weighting method, analytic hierarchy process (AHP) and Delphi method, these are based on the experience of the main methods, the results are greatly influenced by subjective factors, objectivity is relatively weak, persuasion is not high. Rough Sets (RS) theory is applied to analyze and deal with incomplete and uncertain data sets, so as to discover the potential law (knowledge) [3]. Weight allocation method based on RS theory, based on the existing data set, it is not subject to subjective factors and has strong objectivity. On the other hand, domain experts, experts in the experience is precious knowledge resources, it is a valuable to determine the case weight vector. Therefore, on the basis of the above-mentioned objective method, we introduce Fuzzy Analytic Hierarchy Process (FAHP), which is a qualitative and quantitative system analysis method, and further through subjective experience preference factor, seek to establish subject-object fusion mechanism of empowerment, to promote the organization, application and management efficiency of the case of tacit knowledge externalization. 


\section{Complete objective weight allocation based on RS theory}

There are two ways to calculate the weight based on RS theory:A method based on attribute dependence and a method based on conditional entropy (attribute information). The former is to measure the importance of attribute by the influence of the classification of subsets in domain of discourse, the calculation process is intuitive and easy to understand, but the result is relatively rough; The latter measures the importance of attributes from the perspective of the amount of information contained in the condition attributes, and has a finer degree of differentiation, but the process is less intuitive relatively [4-6].

\subsection{Based on the dependence of weights allocation}

Different knowledge on the theory of domain classification ability is different, the weight difference leads to its important degree. Different knowledge on the domain of the classification ability is different, leading to its important degree difference namely weight difference.

The known knowledge expression system $K S=(U, A, V, f)$, among them, $U$ is nonempty finite domains of knowledge expression system, $A$ is attribute set of knowledge expression system, and $A=C \bigcup D ; f$ is assignment function, determines the attribute value of each object, namely $f(A \rightarrow V)$.Decision attribute (set) $D$ completely depends on the attribute sets(C) that composed of multiple condition attributes $\left(a_{1}, a_{2}, \ldots, a_{n}\right)$; However, its dependence on each condition attribute is different, so it can calculate the importance of each condition attribute. Its basic rule is: According to the knowledge representation system, two-dimensional decision table is established in order to eliminate each condition attributes, calculate out before and after decision attribute (set) of the dependence of condition attribute set. The greater the dependency change, the more important the conditional attribute is relative to the decision attribute(set), the higher the weight; On the contrary, the lower.

Conversely, the solving steps are as follows:

(1) Collecting the original data of the evaluation object, discretizing the continuity data and establishing the twodimensional decision table.

(2) Calculate D's C domain $\operatorname{pos}_{C}(D)$, on the basis of the calculation $D$ for the condition attribute set C's dependence:

$$
\gamma_{C}(D)=\frac{\left|\operatorname{pos}_{C}(D)\right|}{|U|}
$$

(3) The new positive domain $\operatorname{pos}_{C-a_{i}}(D)$ is calculated after the conditional attribute $a_{i}$ is removed, on this basis, the dependence on the new conditional attribute set $\left(C-a_{i}\right)$ is calculated:

$$
\gamma_{C-a_{i}}(D)=\frac{\left|\operatorname{pos}_{C-a_{i}}(D)\right|}{|U|}
$$

(4) Calculate the importance of attribute $a_{i}$ to D: $\sigma\left(a_{i}\right)=\gamma_{C-a_{i}}(D)-\gamma_{C}(D)$, and then perform step (3) again, until the condition attribute is overwritten.

(5) Normalization for the whole $\sigma\left(a_{i}\right)$, namely, the weight of condition attributes $a_{i}$ :

$$
\begin{aligned}
& \omega_{1}\left(a_{i}\right)=\frac{\sigma\left(a_{i}\right)}{\sum_{i=1}^{n} \sigma\left(a_{i}\right)} \\
& \omega_{1}\left(a_{i}\right)=\frac{\sigma\left(a_{i}\right)}{\sum_{i=1}^{n} \sigma\left(a_{i}\right)}
\end{aligned}
$$

\subsection{Weight assignment based on conditional entropy}

Entropy is a measure of uncertainty or disorder state, the entropy can be used to judge the degree of discretization of some aspect. The greater the degree of discretization, the greater the effect on the evaluation of case view, ie, the higher the weight [7].Different condition attributes $a \in C$ for decision attribute (set) $D$ provide different information (negative entropy) to decision attributes (sets). When a conditional attribute is added to the conditional attribute set, the amount of information provided by conditional attribute set to decision attribute (set) has changed, showing the 
reduction degree of uncertainty; The greater the amount of information provided, the greater the uncertainty reduction, indicating that the more important condition attributes.

The idea of weighting based on information view is as follows: according to the knowledge representation system, the two-dimensional decision table about evaluation object is established, and the conditional entropy of C and D is calculated [8].Then, the condition attributes are removed in turn and the conditional entropy is recalculated. Finally, according to the degree of change of conditional entropy, we obtain the importance degree of each condition attribute relative to decision attribute (set).

A knowledge representation system $K S=(U, A, V, f)$,among them $A=C \cup D$,

$C=\left\{a_{1}, a_{2}, \ldots, a_{n}\right\}$ is conditional attribute set, $D$ is decision attribute (set), the information weight calculation procedure is as follows:

(1) Collecting the original data of the evaluation objects, discretizing the continuity data and establishing the twodimensional decision table.

(2) The conditional entropy of $D$ with respect to $C$ is calculated $H(D \mid C)$;

(3) Remove the conditional attribute ${ }^{a_{i}}$ in turn, calculating the conditional entropy $H\left(D \mid C-a_{i}\right)$ of $D_{\text {with respect }}$ to $C-a_{i}$.

(4) Calculate the importance of condition attribute ${ }^{a_{i}}$ to decision attribute D:

$\operatorname{Sig}\left(a_{i}, C, D\right)=H\left(D \mid C-a_{i}\right)-H(D \mid C)$

,and perform step (3) again, until the condition attribute is overwritten.

(5) Normalization for the whole $\operatorname{Sig}\left(a_{i}, C, D\right)$,namely, the weight of condition attributes $a_{i}$ :

$$
\omega_{2}\left(a_{i}\right)=\frac{\operatorname{Sig}\left(a_{i}, C, D\right)}{\sum_{i=1}^{n} \operatorname{Sig}\left(a_{i}, C, D\right)}
$$

\subsection{Information concept weight allocation}

Through the foregoing two methods to calculate the weight of the objectivity of the results are good, but if the result will be the only two methods simply count up and normalized processing, the phenomenon of "large number eat decimals" may occur, not rigorous. Taking into account the characteristics of both, this paper introduces the coordination factor $\lambda$ to take into account the advantages of two, and in practice according to the application of demand characteristics of flexible adjustment in order to calculate the benefits and understandable results to find a balance.

Thus, based on the above-mentioned idea and the results of the two methods, the conditional attribute of $a_{i}$ of the complete RS weight is:

$$
\omega_{R S}\left(a_{i}\right)=\lambda \omega_{1}\left(a_{i}\right)+(1-\lambda) \omega_{2}\left(a_{i}\right)
$$

\section{Based on FAHP of case of quantificating attribute subjective weight configuration}

Analytic Hierarchy Process (AHP) is a combination of qualitative and quantitative system analysis method, is suitable for the complex structure, much decision criteria and decision problem is not easy to quantify. AHP quantitative characterization of the inference process, to avoid the problem of decision-making complex and more programs caused by the logic of decision-makers reasoning errors a certain extent [9-11];However, this method also have shortcomings as following:(1) It requires decision-makers to determine the value of the project scale, but in practice decision-makers subjective judgments are ambiguous, the determination of the value scale is difficult to eliminate due to the impact of subjective judgments $[12,13] .(2)$ When the order of the AHP judgment matrix is high, it is difficult to check its consistency; When the consistency of the judgment matrix can't be met,need to repeat adjustment and testing, exhausting process, lower efficiency [14].

The feasible way to deal with the aforementioned shortcomings is:combining the AHP method with the fuzzy theory, it fully integrates the fuzziness of the decision-maker's thinking, makes the judgment matrix more reasonable and easier to pass the consistency test, that is fuzzy analytic hierarchy process (FAHP) [15, 16].It constructs a fuzzy judgment matrix based on fuzzy numbers (such as triangular fuzzy number, trapezoidal fuzzy number,type of L-R fuzzy number, etc.).The triangular fuzzy numbers represent the fuzzy thinking of the decision maker in the form of triples $(l, m, u)$, which is more practical and the method is simple and easy to operate.Among them, $l, m, u$ is the the lower bound, median and upper bound of the fuzzy number respectively.Under normal circumstances, meet $l<m<u$; when 
$l=m=u$, the fuzzy number evolves to an exact value;Besides, $(u-l) /(l+m+u)$ the larger the value, the more fuzzy the fuzzy number.

Based on Fuzzy Theory,the fuzzy scale takes values within[0,1],the rules of the values are shown in Table 1.

Table 1. Fuzzy scale and its meaning.

\begin{tabular}{cc}
\hline Scale value $C_{i j}$ & meaning \\
\hline 0.1 & $\begin{array}{c}\text { Object } \mathrm{j} \text { is more absolutely important than object } \mathrm{i} \\
\text { Object } \mathrm{j} \text { is more strongly important than } \\
\text { object } \mathrm{i}\end{array}$ \\
0.2 & $\begin{array}{c}\text { Object } \mathrm{j} \text { is more obviously important than } \\
\text { object } \mathrm{i}\end{array}$ \\
0.4 & Object $\mathrm{j}$ is slightly more important than \\
object $\mathrm{i}$
\end{tabular}

$0.5 \quad$ Object $\mathrm{j}$ and $\mathrm{I}$ are equally important

$0.6 \quad$ Object $\mathrm{i}$ is slightly more important than object $\mathrm{j}$

$0.7 \quad$ Object $i$ is more obviously important than object $\mathrm{j}$

Object $i$ is more strongly important than object $\mathrm{j}$

$0.9 \quad$ Object $\mathrm{i}$ is more absolutely important than object $\mathrm{j}$

Definition 1:If all elements meet $0 \leq r_{i j} \leq 1$ of square matrix $R=\left(r_{i j}\right)_{n \times n}$,it is the fuzzy matrix.

Definition 2:If all elements meet $r_{i j}+r_{j i}=1$ of fuzzy matrix $R=\left(r_{i j}\right)_{n \times n}$, it is complementary.

Definition 3:If all elements meet $r_{i j}=r_{i k}-r_{j k}+0.5$ of fuzzy complementary phalanx $R=\left(r_{i j}\right)_{n \times n}$,it is consistent array.

Assuming that there are $k$ experts to evaluate the importance of the $n$ properties to be judged by comparing the two judgments, FAHP steps are as follows:

(1) Comparison of two pairs of properties to be evaluated, a fuzzy complementary judgment matrix $R=\left(r_{i j}\right)_{n \times n}$ is established based on triangular fuzzy numbers.

$$
R=\left[\begin{array}{cccc}
\left(l_{11}, m_{11}, u_{11}\right) & \left(l_{12}, m_{12}, u_{12}\right) & \cdots & \left(l_{1 n}, m_{1 n}, u_{1 n}\right) \\
\left(l_{21}, m_{21}, u_{21}\right) & \left(l_{22}, m_{22}, u_{11}\right) & \cdots & \left(l_{2 n}, m_{2 n}, u_{2 n}\right) \\
\vdots & \vdots & \vdots & \vdots \\
\left(l_{n 1}, m_{n 1}, u_{n 1}\right) & \left(l_{n 2}, m_{n 2}, u_{n 2}\right) & \cdots & \left(l_{n n}, m_{n n}, u_{n n}\right)
\end{array}\right]
$$

In $R=\left(r_{i j}\right)_{n \times n}, l_{i i}=m_{i i}=u_{i i}=0.5, l_{i j}+u_{j i}=1, u_{i j}+l_{j i}=1$.

(2) On the basis of,calculating probability matrix $B=\left(b_{i j}\right)_{n \times n}$ and fuzzy evaluation matrix $S=\left(s_{i j}\right)_{n \times n}$ [14]:

$$
\begin{gathered}
b_{i j}=\frac{l_{i j}+4 m_{i j}+u_{i j}}{6}, \\
s_{i j}=1-\frac{u_{i j}-l_{i j}}{2\left(l_{i j}+m_{i j}+u_{i j}\right)} .
\end{gathered}
$$

Among them,the ${ }^{S_{i j}}$ smaller the value of $S$, the larger the fuzzy interval, the fuzziness of the fuzzy number is stronger and the confidence level is lower.

(3) The aforementioned matrix is multiplied $t_{i j}=b_{i j} s_{i j}$,to adjust it to obtain complementary and fuzzy judgment matrix $R^{\prime}=\left(r_{i j}^{\prime}\right)_{n \times n}$. Among them:

$$
r_{i j}^{\prime}=\frac{1}{2}\left(1+t_{i j}-t_{j i}\right)
$$


(4)Adjust $R^{\prime}=\left(r_{i j}^{\prime}\right)_{n \times n}$ for the consistency matrix $R^{\prime \prime}=\left(r_{i j}^{\prime \prime}\right)_{n \times n}$, among them:

$$
r_{i j}^{\prime \prime}=\frac{r_{i}^{\prime}-r_{j}^{\prime}}{2(n-1)}+0.5, r_{i}^{\prime}=\sum_{a=1}^{n} r_{i a}^{\prime} .
$$

(5)Weighting average fuzzy judgment matrix for each evaluation expert and to get the comprehensive matrix $\tilde{R}=\left(\tilde{r_{i j}}\right)_{n \times n}$,

$$
\tilde{r}_{i j}=\frac{1}{k} \sum_{p=1}^{k} r_{i j}^{\prime \prime}(p)
$$

Among them: $r_{i j}(p)$ represents the first evaluation expert's in $R^{\prime \prime}$ corresponding evaluation elements.

(6)The final weight vector is obtained by the following equation $\omega_{F A H P}=\left(\omega_{F A H P}^{1}, \omega_{F A H P}^{2}, \cdots, \omega_{F A H P}^{n}\right)$ :

$$
\omega_{\text {FAHP }}^{i}=\frac{\sum_{j=1}^{n} \tilde{r}_{i j}+\frac{n}{2}-1}{n(n-1)}
$$

The above algorithm is based on the fuzzy consistent matrix expansion process, which avoids the process of consistency and continuation of the traditional AHP method, to ensure the efficiency of solving.

\section{Case attributes RS-FAHP comprehensive weight configuration}

Based on the implicit knowledge explicit case model, the condition attribute set and decision attribute are established;Importing tacit knowledge in the explicit case database through discretizing of case data, and the knowledge expression system $K S=(U, A, V, f)$ is established.Thereinto, $A=C \cup D, C=\left\{a_{1}, a_{2}, \ldots, a_{n}\right\}$ is conditional attribute set, $D$ is decision attribute.

Using $\omega_{i} \quad(i=1,2, \cdots, n)$ to express RS-FAHP comprehensive weight,Obviously $\omega_{R S}^{i}, \omega_{F A H P}^{i}$ should meet the following conditions:

$$
\sum_{i=1}^{n} \omega_{R S}^{i}=\sum_{i=1}^{n} \omega_{F A H P}^{i}=\sum_{i=1}^{n} \omega_{i}=1,
$$

$0 \leq \omega_{R S}^{i} \leq 1,0 \leq \omega_{F A H P}^{i} \leq 1,0 \leq \omega_{i} \leq 1$.

The fusion factor $\mathcal{E}$ (meet $0 \leq \varepsilon \leq 1$ ) was introduced,establish the optimal model:

$$
\min \left\{\sum_{i=1}^{n}\left[\varepsilon\left(\frac{1}{2}\left(\omega_{i}-\omega_{R S}^{i}\right)^{2}\right)+(1-\varepsilon)\left(\frac{1}{2}\left(\omega_{i}-\omega_{F A H P}^{i}\right)^{2}\right)\right]\right\}
$$

To solve the above equation, Lagrange function:

$$
L\left(\omega_{i}, \lambda\right)=\sum_{i=1}^{n}\left[\varepsilon\left(\frac{1}{2}\left(\omega_{i}-\omega_{R S}^{i}\right)^{2}+(1-\varepsilon)\left(\frac{1}{2}\left(\omega_{i}-\omega_{F A H P}^{i}\right)^{2}\right)\right]+\lambda\left(\sum_{i=1}^{n} \omega_{i}-1\right)\right.
$$

Find the partial derivatives of $\omega_{i}, \lambda$ respectively, and the other

$$
\begin{gathered}
\frac{\partial L}{\partial \omega_{i}}=0 \frac{\partial L}{\partial \lambda}=0 \\
\left\{\begin{array}{l}
\varepsilon\left(\omega_{i}-\omega_{R S}^{i}\right)+(1-\varepsilon)\left(\omega_{i}-\omega_{F A H P}^{i}\right)=0 \\
\sum_{i=1}^{n} \omega_{i}-1=0
\end{array}\right.
\end{gathered}
$$

Furthermore: $\omega_{i}=\varepsilon \omega_{R S}^{i}+(1-\varepsilon) \omega_{F A H P}^{i}$.It can be seen that the fusion factor contains the subjective experience preference of the evaluation subject, the smaller the value is, the stronger the subjective preference is.

\section{Example analysis}


Assuming that an explicit case model with implicit knowledge:the condition section is composed of four aspects, and the decision section is composed of one aspect,respectively for $C=\left\{a_{1}, a_{2}, a_{3}, a_{4}\right\}$ and $D=\{d\}$. The case subspace in this mode is 10 , after case data were imported and discretized, the knowledge expression system $K S=(U, A, V, f)$ was established,as shown in table 2.

Table 2. Knowledge representation system based on explicit case subspace of tacit knowledge.

\begin{tabular}{cccccc}
\hline $\mathrm{U}$ & $\mathrm{a}_{1}$ & $\mathrm{a}_{2}$ & $\mathrm{a}_{3}$ & $\mathrm{a}_{4}$ & $\mathrm{~d}$ \\
\hline $\mathrm{x}_{1}$ & 1 & 1 & 0 & 0 & 1 \\
$\mathrm{x}_{2}$ & 1 & 0 & 0 & 0 & 1 \\
$\mathrm{x}_{3}$ & 0 & 0 & 0 & 0 & 0 \\
$\mathrm{x}_{4}$ & 1 & 1 & 0 & 1 & 0 \\
$\mathrm{x}_{5}$ & 1 & 2 & 0 & 1 & 2 \\
$\mathrm{x}_{6}$ & 2 & 2 & 0 & 2 & 2 \\
$\mathrm{x}_{7}$ & 2 & 2 & 2 & 2 & 2 \\
$\mathrm{x}_{8}$ & 1 & 2 & 4 & 1 & 3 \\
$\mathrm{x}_{9}$ & 2 & 2 & 4 & 2 & 3 \\
$\mathrm{x}_{10}$ & 2 & 1 & 0 & 0 & 3 \\
\hline
\end{tabular}

In order to establish the case view of the case model, the calculation of the case is based on the RS-FAHP method. Firstly,weight algorithm based on degree of dependence $\omega_{1}\left(a_{i}\right)$ (among $\left.i=1,2,3,4\right)$, calculated as follows:

$U / d=\left\{\left\{x_{1}, x_{2}\right\},\left\{x_{3}, x_{4}\right\},\left\{x_{5}, x_{6}, x_{7}\right\},\left\{x_{8}, x_{9}, x_{10}\right\}\right\} U / C=\left\{\left\{x_{1}\right\},\left\{x_{2}\right\},\left\{x_{3}\right\},\left\{x_{4}\right\},\left\{x_{5}\right\},\left\{x_{6}\right\},\left\{x_{7}\right\},\left\{x_{8}\right\},\left\{x_{9}\right\},\left\{x_{10}\right\}\right\}$

$U /\left\{C-a_{1}\right\}=\left\{\left\{x_{1}, x_{10}\right\},\left\{x_{2}, x_{3}\right\},\left\{x_{4}\right\},\left\{x_{5}\right\},\left\{x_{6}\right\},\left\{x_{7}\right\},\left\{x_{8}\right\},\left\{x_{9}\right\}\right\} U /\left\{C-a_{2}\right\}=\left\{\left\{x_{1}, x_{2}\right\},\left\{x_{3}\right\},\left\{x_{4}, x_{5}\right\},\left\{x_{6}\right\},\left\{x_{7}\right\},\left\{x_{8}\right\},\left\{x_{9}\right\},\left\{x_{10}\right\}\right\}$

$U /\left\{C-a_{3}\right\}=\left\{\left\{x_{1}\right\},\left\{x_{2}\right\},\left\{x_{3}\right\},\left\{x_{4}\right\},\left\{x_{5}, x_{8}\right\},\left\{x_{6}, x_{7}, x_{9}\right\},\left\{x_{10}\right\}\right\} U /\left\{C-a_{4}\right\}=\left\{\left\{x_{1}, x_{4}\right\},\left\{x_{2}\right\},\left\{x_{3}\right\},\left\{x_{5}\right\},\left\{x_{6}\right\},\left\{x_{7}\right\},\left\{x_{8}\right\},\left\{x_{9}\right\},\left\{x_{10}\right\}\right\}$

The results are as follows:

$$
\begin{gathered}
\gamma_{C}(d)=1, \gamma_{C-a_{1}}(d)=0.7 \\
\gamma_{C-a_{2}}(d)=0.8, \gamma_{C-a_{3}}(d)=0.5 \\
\gamma_{C-a_{4}}(d)=0.8 .
\end{gathered}
$$

Thus, the importance of each attribute can be obtained as:

$$
\begin{aligned}
& \sigma\left(a_{1}\right)=0.3, \sigma\left(a_{2}\right)=0.2, \\
& \sigma\left(a_{3}\right)=0.5, \sigma\left(a_{4}\right)=0.2 .
\end{aligned}
$$

The above results are normalized, and the weight of each attribute based on dependency is as follows:

$$
\begin{aligned}
& \omega_{1}\left(a_{1}\right)=0.2500, \omega_{1}\left(a_{2}\right)=0.1667, \\
& \omega_{1}\left(a_{3}\right)=0.4166, \omega_{1}\left(a_{4}\right)=0.1667 .
\end{aligned}
$$

Secondly, Weight algorithm based on conditional entropy $\omega_{2}\left(a_{i}\right)$.

$$
\begin{gathered}
H(d \mid C)=0, \quad H\left(d \mid C-a_{1}\right)=0.4 \\
H\left(d \mid C-a_{2}\right)=0.2, \quad H\left(d \mid C-a_{3}\right)=0.4755 \\
H\left(d \mid C-a_{4}\right)=0.2
\end{gathered}
$$

According to $\operatorname{Sig}\left(a_{i}, C, D\right)$, further calculation of the importance of each attribute is:

$$
\begin{aligned}
& \operatorname{Sig}\left(a_{1}, C, D\right)=0.4, \quad \operatorname{Sig}\left(a_{2}, C, D\right)=0.2, \\
& \operatorname{Sig}\left(a_{3}, C, D\right)=0.4755, \operatorname{Sig}\left(a_{4}, C, D\right)=0.2
\end{aligned}
$$

The above results are normalized, and the weight of each attribute based on conditional entropy is as follows:

$$
\begin{aligned}
& \omega_{2}\left(a_{1}\right)=0.3136, \omega_{2}\left(a_{2}\right)=0.1568, \\
& \omega_{2}\left(a_{3}\right)=0.3728, \omega_{2}\left(a_{4}\right)=0.1568 .
\end{aligned}
$$

Based on the above results, considering the high degree of differentiation based on conditional entropy, taking into coordination factor $\lambda=0.45$, according to 1.3 Section information concept weight allocation, the complete objective weight of each attribute based on RS theory is:

$$
\omega_{R S}\left(a_{1}\right)=0.2850, \omega_{R S}\left(a_{2}\right)=0.1613,
$$




$$
\omega_{R S}\left(a_{3}\right)=0.3924, \omega_{R S}\left(a_{4}\right)=0.1613 .
$$

Then,based on the aforementioned FAHP algorithm to solve the subjective weight of each attribute of the case.In this case, the importance of each case attribute based on triangular fuzzy number is given by three experts $(k=3)$ in the field,the result is as follows:

$$
\begin{aligned}
& R_{1}=\left[\begin{array}{llll}
(0.5,0.5,0.5) & (0.5,0.6,0.7) & (0.3,0.4,0.5) & (0.5,0.6,0.7) \\
(0.3,0.4,0.5) & (0.5,0.5,0.5) & (0.2,0.3,0.4) & (0.45,0.5,0.55) \\
(0.5,0.6,0.7) & (0.6,0.7,0.8) & (0.5,0.5,0.5) & (0.65,0.7,0.75) \\
(0.3,0.4,0.5) & (0.45,0.5,0.55) & (0.25,0.3,0.35) & (0.5,0.5,0.5)
\end{array}\right] \\
& R_{2}=\left[\begin{array}{cccc}
(0.5,0.5,0.5) & (0.55,0.6,0.65) & (0.35,0.4,0.45) & (0.5,0.6,0.7) \\
(0.35,0.4,0.45) & (0.5,0.5,0.5) & (0.25,0.3,0.35) & (0.45,0.5,0.55) \\
(0.55,0.6,0.65) & (0.65,0.7,0.75) & (0.5,0.5,0.5) & (0.65,0.7,0.75) \\
(0.3,0.4,0.5) & (0.45,0.5,0.55) & (0.25,0.3,0.35) & (0.5,0.5,0.5)
\end{array}\right] \\
& R_{3}=\left[\begin{array}{cccc}
(0.5,0.5,0.5) & (0.65,0.7,0.75) & (0.35,0.4,0.45) & (0.5,0.6,0.7) \\
(0.25,0.3,0.35) & (0.5,0.5,0.5) & (0.25,0.3,0.35) & (0.4,0.5,0.6) \\
(0.55,0.6,0.65) & (0.65,0.7,0.75) & (0.5,0.5,0.5) & (0.65,0.7,0.75) \\
(0.3,0.4,0.5) & (0.4,0.5,0.6) & (0.25,0.3,0.35) & (0.5,0.5,0.5)
\end{array}\right]
\end{aligned}
$$

On the basis of this, the probability matrix and the fuzzy judgment matrix are calculated as follows:

$$
\begin{aligned}
& B_{1}=B_{2}=\left[\begin{array}{llll}
0.5 & 0.6 & 0.4 & 0.6 \\
0.4 & 0.5 & 0.3 & 0.5 \\
0.6 & 0.7 & 0.5 & 0.7 \\
0.4 & 0.5 & 0.3 & 0.5
\end{array}\right] \\
& B_{3}=\left[\begin{array}{llll}
0.5 & 0.7 & 0.4 & 0.6 \\
0.3 & 0.5 & 0.3 & 0.5 \\
0.6 & 0.7 & 0.5 & 0.7 \\
0.4 & 0.5 & 0.3 & 0.5
\end{array}\right] \\
& S_{1}=\left[\begin{array}{cccc}
1 & 0.944 & 0.917 & 0.944 \\
0.917 & 1 & 0.889 & 0.967 \\
0.944 & 0.952 & 1 & 0.976 \\
0.917 & 0.967 & 0.944 & 1
\end{array}\right] \\
& S_{2}=\left[\begin{array}{cccc}
1 & 0.972 & 0.958 & 0.944 \\
0.958 & 1 & 0.944 & 0.967 \\
0.972 & 0.976 & 1 & 0.976 \\
0.917 & 0.967 & 0.944 & 1
\end{array}\right] \\
& S_{3}=\left[\begin{array}{cccc}
1 & 0.976 & 0.958 & 0.944 \\
0.944 & 1 & 0.944 & 0.933 \\
0.972 & 0.976 & 1 & 0.976 \\
0.917 & 0.933 & 0.944 & 1
\end{array}\right]
\end{aligned}
$$

The corresponding probability matrix is multiplied by the fuzzy evaluation matrix, and the computation of the complementary fuzzy judgment matrix $R^{\prime}=\left(r_{i j}^{\prime}\right)_{n \times n}$ is as follows:

$$
R_{1}^{\prime}=\left[\begin{array}{cccc}
0.5 & 0.5998 & 0.4002 & 0.5998 \\
0.4002 & 0.5 & 0.3002 & 0.5 \\
0.5998 & 0.6998 & 0.5 & 0.7 \\
0.4002 & 0.5 & 0.3 & 0.5
\end{array}\right]
$$




$$
\begin{aligned}
R_{2}^{\prime} & =\left[\begin{array}{cccc}
0.5 & 0.6 & 0.4 & 0.5998 \\
0.4 & 0.5 & 0.3 & 0.5 \\
0.6 & 0.7 & 0.5 & 0.7 \\
0.4002 & 0.5 & 0.3 & 0.5
\end{array}\right] \\
R_{3}^{\prime} & =\left[\begin{array}{cccc}
0.5 & 0.7 & 0.4 & 0.5998 \\
0.3 & 0.5 & 0.3 & 0.5 \\
0.6 & 0.7 & 0.5 & 0.7 \\
0.4002 & 0.5 & 0.3 & 0.5
\end{array}\right]
\end{aligned}
$$

Further, the fuzzy complementary matrix $R^{\prime}=\left(r_{i j}^{\prime}\right)_{n \times n}$ is adjusted to be fuzzy consistency matrix $R^{\prime \prime}=\left(r_{i j}^{\prime \prime}\right)_{n \times n}$ :

$$
\begin{aligned}
R_{1}^{\prime \prime} & =\left[\begin{array}{cccc}
0.5 & 0.4334 & 0.5666 & 0.4334 \\
0.5666 & 0.5 & 0.6332 & 0.5 \\
0.4334 & 0.3668 & 0.5 & 0.3668 \\
0.5666 & 0.5 & 0.6332 & 0.5
\end{array}\right] \\
R_{2}^{\prime \prime} & =\left[\begin{array}{cccc}
0.5 & 0.4334 & 0.5667 & 0.4334 \\
0.5666 & 0.5 & 0.6333 & 0.5 \\
0.4333 & 0.3667 & 0.5 & 0.3667 \\
0.5666 & 0.5 & 0.6333 & 0.5
\end{array}\right] \\
R_{3}^{\prime \prime} & =\left[\begin{array}{cccc}
0.5 & 0.4067 & 0.5567 & 0.4234 \\
0.5933 & 0.5 & 0.65 & 0.5167 \\
0.4433 & 0.35 & 0.5 & 0.3667 \\
0.5766 & 0.4833 & 0.6333 & 0.5
\end{array}\right]
\end{aligned}
$$

On the basis of this, the matrix formula $\tilde{R}=\left(\tilde{r}_{i j}\right)_{n \times n}$ is obtained based on the fuzzy consistent complementary judgment matrix:

$$
\tilde{R}=\left[\begin{array}{cccc}
0.5 & 0.4235 & 0.5666 & 0.4334 \\
0.5755 & 0.5 & 0.6388 & 0.5056 \\
0.4367 & 0.3612 & 0.5 & 0.3667 \\
0.5699 & 0.4944 & 0.6333 & 0.5
\end{array}\right]
$$

At this point, through the calculation method of the final weight vector $\omega=\left(\omega_{1}, \omega_{2}, \cdots, \omega_{n}\right)$, the weight vector of the algorithm based on FAHP algorithm is:

$$
\omega=(0.2751,0.1859,0.3524,0,1866)
$$

Finally,in the "outstanding objectivity, taking into account the subjective experience" principle, take the fusion factor $\varepsilon=0.65$,according to the algorithm shown in Section 3 ,the results are as follows:

$$
\omega=(0.2751,0.1859,0.3524,0.1866) \text {. }
$$

\section{Conclusion}

In the era of knowledge economy, knowledge management, especially for the effective management of tacit knowledge is to enhance the core competence of the organization, to achieve an important foundation for sustainable development.In this paper, the idea of CBR system is introduced, which is based on the external of tacit knowledge, the RS system is based on the two kinds of weight calculation method, which is based on the attribute dependence and conditional entropy, at the same time, by taking into account the valuable experience of experts in the field of FAHP, the RS-FAHP weight configuration of the subjective and objective fusion of the explicit case view of tacit knowledge is realized.Compared with the traditional subjective experience empowerment method, this method is more rigorous data base and reasoning process, strong objectivity, good interpretability; compared with pure objective method, this method is to fully mix the subjective experience of experts in the field, not only realize the flexible complement to objectively, but also improve the acceptance level of empowerment results. 


\section{Acknowledgement}

This work is supported by the Excellent Young Teacher Development Fund Project of Zhengzhou University (Foundation No. 2015SKYQ15).

\section{References}

1. Shah Bano, John Taylor. Universities and the knowledge-based economy: perceptions from a developing country. Higher Education Research \& Development,34,242-255(2015).

2. Ahrweiler Petra1, Pyka Andreas, Gilbert Nigel. A new model for university-industry links in knowledge-based economies. Journal of Product Innovation Management, 8,218-235(2011).

3. Zdzisław Pawlak. Rough Sets.International Journal of Computer and Information Sciences, 11,341- 356(1982).

4. Zhang Jianhua. Multi-Case Derivational Adaptation Mechanism of KML-Case for Knowledge Management.JournalofSystems\& Management, 23,845-853(2014).

5. Zhu canhong, Chen nenghua.Improvement of Method of Determining Entropy Weight of Conditional Information in Rough Set.Statistics \& Decision,8,154-156(2011).

6. Bao XinZhong,Liu cheng.A New Method of Ascertaining Attribute Weight Based on Rough Sets Theory.Chinese Journal of Management,6,729-736(2009).

7. Zhang Jianhua.Electronic commerce knowledge management. Beijing: Chinese Science Publishing,(2010).

8. Zhai JunChuang, Che WeiWei.A Spam Filtering Algorithm Based on Conditional Entropy. JiSuanJi Yu XianDaiHua,2,129-132(2014).

9. Li ChunHao,Sun YongHe,Jia YanHui.Analytic Hierarchy Process Based on Variable Weights.Systems Engineering-Theory \&Practice, 30,723-731(2010).

10. Liu Jun,Li XiaoNan.Handover Algorithm for WLAN/Cellular Networks with Analytic Hierarchy Process.Journal on Communications, 34,65-72(2013).

11. Cai Yan,Wu Min,Zhou JinNi.AHP-based Fuzzy Multiple Criteria Optimization Methods for Storage Location Choice.Journal of Hunan University(Natural Sciences),40,103-108(2013).

12. Thomas L. Saaty. Modeling unstructured decision problems-the theory of analytical hierarchies. Mathematics and Computers in Simulation,47,147-158(1978).

13. Wang HuaJi,Zong HuaFu,Guan Xin.Method of Determining Weights Subjective Evaluation Indexes for Car Handing and stability Based on Fuzzy Analytic Hierarchy process.Journal of Mechanical Engineering,47,8390(2011).

14. Cheng Kai.An Evaluation Study on Application Condition of Residential PCa Technology on Fuzzy Analytic Hierarchy Process.HangZhou:ZhengJang University,(2012).

15. Lei Lei.Evaluation of Critical Success Factors of Industry Based on FAHP and Fuzzy Delphi Method.Statistics and Decision Making, 15,44-47(2014).

16. Tan BaiYing.The Research of Scale System and Ranking Method of FAHP.NanNing:GuangXi University,(2005). 